\title{
Plasma Neuronal Injury Marker Neurofilament in the First Days of Life in Preterm and Term born Infants
}

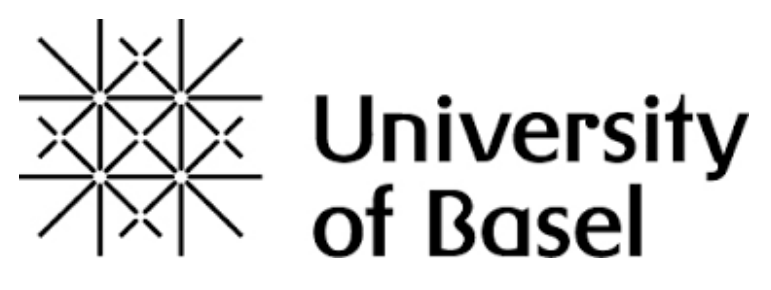

\author{
A. Depoorter ${ }^{1}$, R. Neumann'ㄹ, P. Weber ${ }^{1}$, J. Kuhle 3 , S. Wellmann² \\ 1'Department of Neuropediatrics and Developmental Medicine, University Children's Hospital Basel (UKBB), University of Basel, Basel, Switzerland \\ 2Department of Neonatology, University Children's Hospital Basel, University of Basel (UKBB), Basel, Switzerland \\ ${ }^{3}$ Neurologic Clinic and Policlinic, Departments of Medicine, Biomedicine and Clinical Research, University Hospital Basel, University of Basel, Basel, \\ Switzerland
}

\section{BACKGROUND AND AIM}

- Neurofilament $(\mathrm{Nf})=$ proteins form the axonal skeleton

- Quantification of Nf proteins in the peripheral blood has been proposed in adults as biomarker for neuronal injury (1)

- Levels of Nf proteins in newborn infants are largely unknown

- Aim: To investigate Nf light chain (NfL) plasma concentrations in preterm and term born infants during the first days of life

\section{METHODS}

This prospective observational study was performed at the University Children's Hospital Basel (UKBB). Neonates ( $n=203$ ) with a median GA of 37.9 (IQR 31.9-39.4) weeks were included.

- Blood samples: 185 umbilical venous blood samples at birth and 39 venous blood samples at day of life (DOL) 3

- Plasma NfL was measured by a highly sensitive single molecule array (Simoa) assay

\section{RESULTS I}

The cohort was divided into a very preterm group (GA $<32$ weeks; $n=52$ ) and a moderate preterm and term (MPT) group ( $G A \geq 32$ weeks; $n=151$ ).

\begin{tabular}{|r|c|c|}
\hline & $\begin{array}{c}\text { Moderate Preterm and } \\
\text { Term }(\geq 32 \text { weeks GA) } \\
\mathrm{n}=151\end{array}$ & $\begin{array}{c}\text { Very preterm } \\
(<32 \text { weeks GA }) \\
\mathrm{n}=52\end{array}$ \\
\hline Neonatal characteristics & & $30.1(28.3-31.3)$ \\
\hline GA (weeks) & $38.3(37.0-40.0)$ & $1360(1063-1463)$ \\
\hline BW (g) & $3270(2710-3630)$ & $25(48.1)$ \\
\hline Sex (male, \%) & $87(57.6)$ & $10(19.2)$ \\
\hline $\mathrm{O}_{2}$ duration (days) & $1(0.7)$ & $4(1-15.8)$ \\
\hline pH umbilical artery & 0 & $7.32(7.29-7.37)$ \\
\hline NEC (\%) & $7.30(7.26-7.33)$ & 0 \\
\hline Sepsis (\%) & 0 & $11(21.2)$ \\
\hline 5-min Apgar & 0 & $7(5.25-8)$ \\
\hline Death (\%) & $9(9-9)$ & $6(11.5)$ \\
\hline Maternal characteristics & 0 & $33(28.3-36.0)$ \\
\hline Age (years) & $32(29-36)$ & $13(25)$ \\
\hline Amniotic infection (\%) & $5(3.3)$ & $20(38.5)$ \\
\hline Preeclampsia (\%) & $16(10.6)$ & $14(26.9)$ \\
\hline PROM (\%) & $14(9.3)$ & $26(50)$ \\
\hline DM (\%): Primary CS & $76(50.3)$ & $21(40.4)$ \\
Secondary CS & $29(19.2)$ & $5(9.6)$ \\
VD & $46(30.5)$ &
\end{tabular}

The median plasma concentration of $\mathrm{NfL}$ at birth $(18.2 \mathrm{pg} / \mathrm{mL}$, IQR 12.8-30.8) was lower compared to DOL $3(50.9 \mathrm{pg} / \mathrm{mL}$, IQR 41.3-100.1, $\mathrm{p}<.001)$. NfL levels were higher in very preterm infants than in the MPT group at birth (median $32.5 \mathrm{pg} / \mathrm{mL}, \mathrm{n}=47$ vs. $15.3 \mathrm{pg} / \mathrm{mL}, \mathrm{n}=138 ; \mathrm{p}<.001$ ), but not at DOL 3 (median 48.5 $\mathrm{pg} / \mathrm{mL}, \mathrm{n}=16$ vs. $51.4 \mathrm{pg} / \mathrm{mL}, \mathrm{n}=23 ; \mathrm{p}=.668)$.

\section{RESULTS II}

NfL levels at birth significantly correlated with BW $(R=-.403$, $\mathrm{p}<.001$, Figure 1), GA ( $\mathrm{R}=-.271, \mathrm{p}<.001), 5-\mathrm{min}$ Apgar $(\mathrm{R}=-.295$, $\mathrm{p}<.001)$ and oxygen duration $(\mathrm{R}=.333, \mathrm{p}<.001)$.

Presence of preeclampsia $(31.0 \mathrm{pg} / \mathrm{mL}$ vs. $16.2, \mathrm{p}<.001)$ and sepsis (32.6 $\mathrm{pg} / \mathrm{mL}$ vs. $17.85, \mathrm{p}=.033$ ) were associated with higher NfL levels.

In the MPT group NfL levels at birth were higher in infants delivered vaginally than by primary or secondary cesarean section (21.8 vs. 13.9 and $14.4 \mathrm{pg} / \mathrm{mL} ; \mathrm{p}=.002$ ) (Figure 2).

Multivariable logistic regression analysis identified as independent predictors of NfL: BW (beta=-.297, $p=.003$ ), delivery mode (beta $=.237, \mathrm{p}=.001$ ) and preeclampsia (beta=.183, $\mathrm{p}=.022)$.
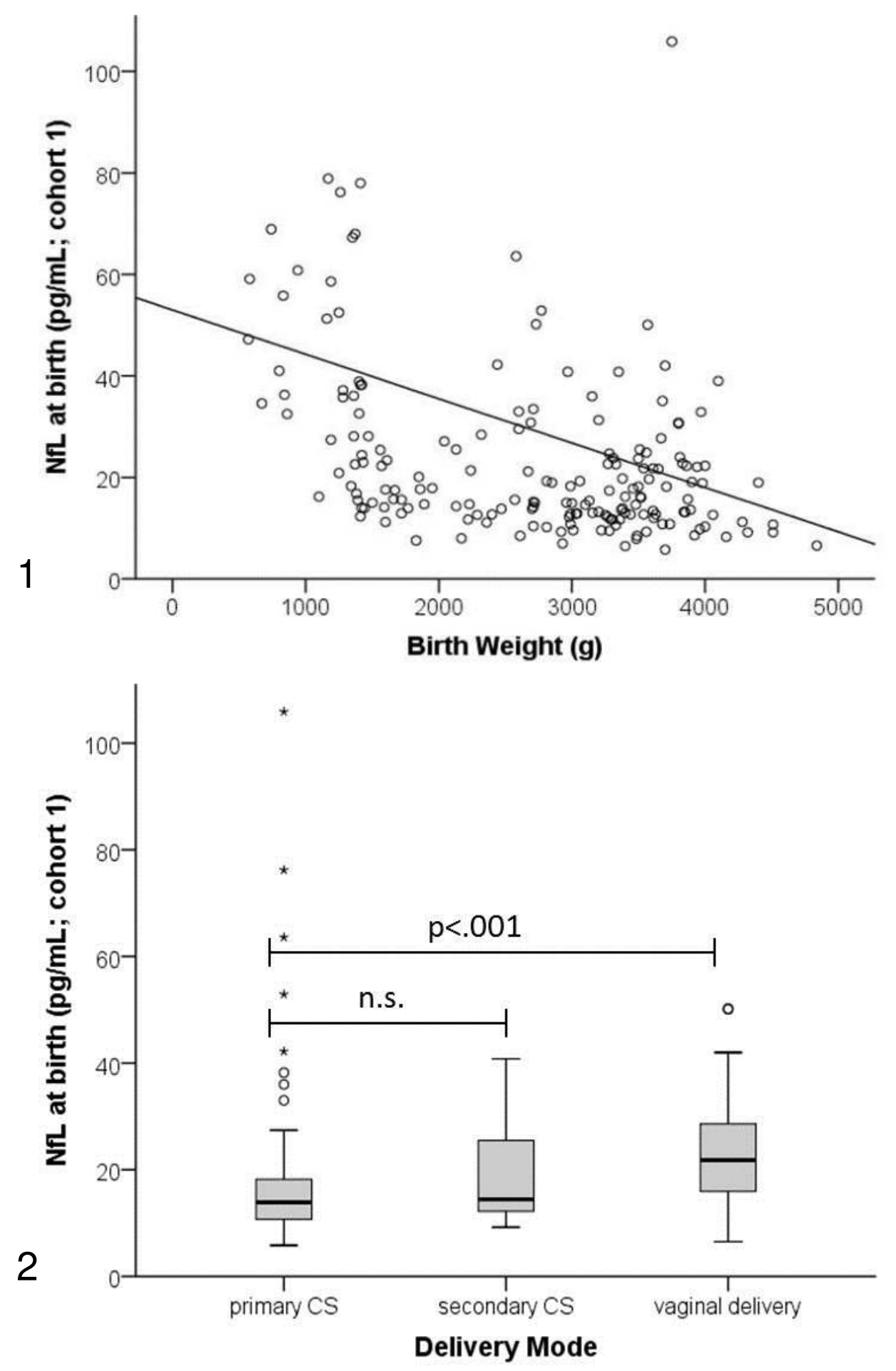

\section{CONCLUSION}

Neonatal NfL levels correlate inversely with maturity and BW, increase during the first days of life and are influenced by maternal factors such as delivery mode and preeclampsia.

1: Yuan A, Rao MV, Veeranna, Nixon RA. Neurofilaments and Neurofilament Proteins in Health and Disease. Cold Spring Harbor perspectives in biology. 2017;9(4). 